\title{
CHANGING EPIDEMIOLOGY AND TRANSMISSIBILITY IN MEASLES INFECTIONS FROM 2009 to 2016, SOUTHERN CHINA
}

K.C. Chong ${ }^{1}$, P. Hu ${ }^{2}$, R. Sun ${ }^{1}$, W. Liang ${ }^{2}$, M. Wang ${ }^{1}$, H. Zheng ${ }^{2}$

${ }^{1}$ The Chinese University of Hong Kong, School of Public Health and Primary Care, Hong Kong.

${ }^{2}$ Center for Disease Control and Prevention of Guangdong Province, Guangzhou, China

\section{Background}

Measles, a highly contagious disease caused by the measles virus, has continuously circulated and caused significant burden of disease globally. To date, measles remained as one of the leading causes of morbidity and mortality among children worldwide and 95\% measles deaths were attributable to countries with low-income and weak health infrastructures. As measles was effectively prevented and controlled by vaccination, China began to use the measles vaccine in the 1960s and raised an expanded immunization program in 1978. Despite several immunization efforts, China saw a resurgence of measles in 2012. It probably due to a change of measles epidemiology over time, particularly before and after the provincial supplementary immunization activities (SIAs). In addition, monitoring of age-specific transmissibility should be conducted for planning adequate disease control strategies.

In Guangdong, a province in Southern China, resurgence of measles incidence in 2013 occurred after the 2009 province-wide and 2010 nation-wide SIAs, and the high prevalence persisted in 2013-2014 despite several smaller-sized SIAs and mop-up campaigns being carried out in some counties. In this study, we aim to assess the changes on measles epidemiology and transmissibility over the study period of 20092016

\section{Method}

The measles data from January 2009 and December 2016 were extracted from the National Infectious Disease Monitoring Information System (NIDMIS) by the Centers for Disease Control and Prevention of Guangdong Province (Guangdong CDC).
Information on patients' demographic background (e.g. age, sex, birth date, district of residence and household registration), date of onset for disease and symptoms, and immunization history was also retrieved from the NIDMIS. The changes on epidemiological characteristics between the 2009-11 and 2012-16, as marked by the end of provincewide SIA and start of resurgences were also compared. The method for estimating the age-specific effective reproduction numbers $(R)$ was developed based on the work by White et al.

\section{Results}

From 2009 to 2016, there were 22,362 cases of measles in Guangdong province. The annual incidence rate ranged between $0.295 / 100,000$ (2011) and 6.64/100,000 (2013) during the study period, and the average annual incidence was 2.67/100,000 (Figure 1). Children cases, especially for infants aged 0-8 months had replaced the 725 years old in having most measles cases during the resurgence after $2012(p<0.01)$. Compared to the 2011 level of 32 cases $(10.3 \%$ of all cases), the number of cases among infants of $0-8$ months old surged to 2306 (32.8\% of all cases) in 2013 and decreased back to $333(26.3 \%)$ in 2016.

Figure 2 presents the estimated age-specific effective reproductive numbers for 7 age groups. The major driving force of the outbreak was children aged 0-6 years (peak $R$ values $>1$ ). Nevertheless, adult cases had an increasing proportion over years in Guangdong and replaced the 0-8 months old infants in being the highest-incidence group in $2016(28.7 \%$ of all cases). Adults aged 26-45 years demonstrated the highest transmission (peak $R$ of 1.24 and 1.20 in 2014 and 2015, respectively)

\section{Discussion}

The findings provide the epidemiological basis for better planning of immunization strategies. Although the provincial SIAs conducted in 2009 and 2010 were able to reduce the transmission rates, higher effective reproductive numbers for children aged under 6 years were observed after 2012, which indicates that the benefits of the SIAs were short-lived. Disease control strategies should target children and adult groups that carry high potential for measles transmission. Enhancing vaccination timeliness for routine-immunization eligible children is also recommended.
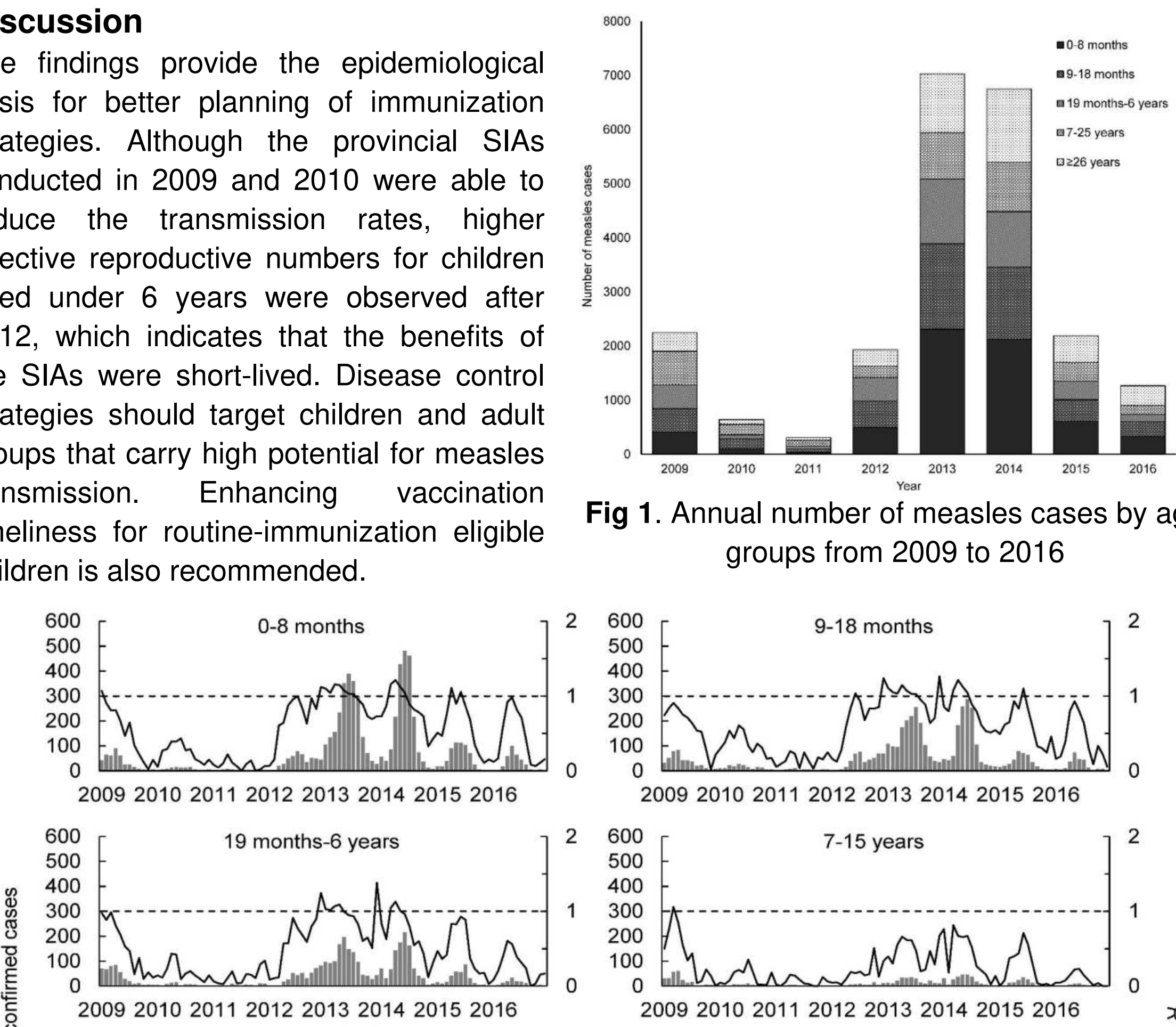

Fig 1. Annual number of measles cases by age groups from 2009 to 2016
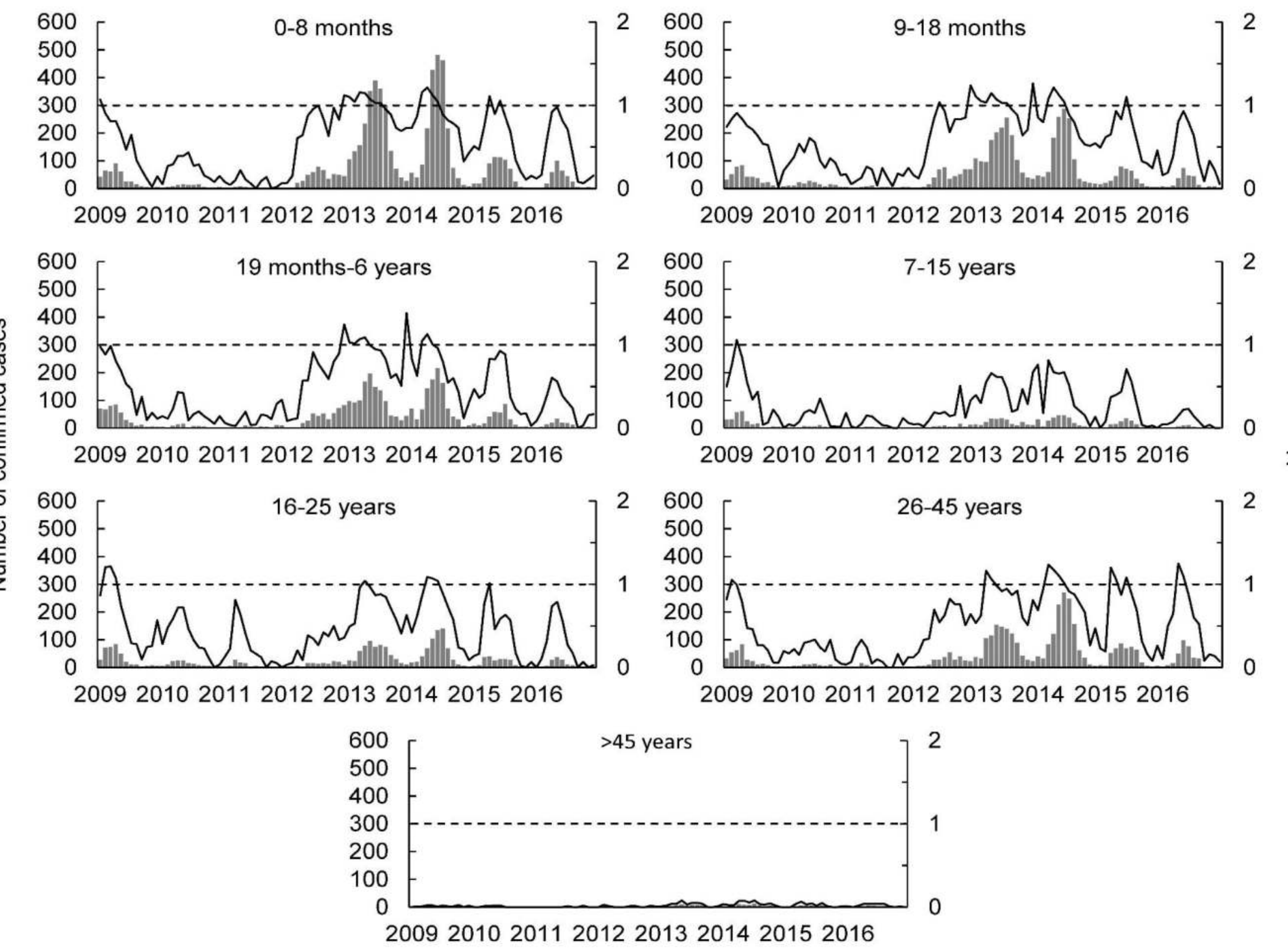

Fig 2. Monthly number of reported cases (histogram) and effective reproductive numbers (solid line) for 7 age groups

Ref: White et al. Determining the dynamics of influenza transmission by age. Emerg Themes Epidemiol. 2014:11:4. 